Revista Brasileira de Agricultura Irrigada v.10, nº.2, p. 522 - 532, 2016

ISSN 1982-7679 (On-line)

Fortaleza, CE, INOVAGRI - http://www.inovagri.org.br

DOI: $10.7127 /$ rbai.v10n200388

Protocolo 388.16 - 06/12/2015 Aprovado em 18/04/2016

\title{
CALIBRAÇÃO DE SONDA ARTESANAL DE USO COM TDR PARA AVALIAÇÃO DE UMIDADE DE SOLOS
}

\author{
Lucas dos Santos Batista ${ }^{1}$, Eugênio Ferreira Coelho ${ }^{2}$, Francisco Adriano de Carvalho \\ Pereira $^{3}$, Mairton Gomes da Silva ${ }^{4}$, Raimundo Rodrigues Gomes Filho ${ }^{5}$, Amanda de \\ Azevedo Gonçalves ${ }^{6}$
}

\begin{abstract}
RESUMO
A Reflectometria no Domínio do Tempo (TDR) tem sido uma ferramenta muito importante para a quantificação do conteúdo da água no solo. Todavia, para utilizar o equipamento há necessidade de calibração para se obter valores acurados de umidade volumétrica, tendo em vista que há diferença entre os tipos de componentes da matriz do solo e na porosidade. Diante do exposto, este trabalho teve como objetivo desenvolver e calibrar sondas de uso em equipamento TDR em diferentes tipos de solos. A calibração foi realizada em condições de laboratório, utilizando amostras de solo acondicionadas em colunas de PVC com volume de 0,0078 m3. As sondas fabricadas artesanalmente com três hastes (espaçadas 0,022 m) e 0,15 $\mathrm{m}$ de comprimento, foram instaladas verticalmente nas colunas de solo, com uma sonda por coluna. Realizaram-se as pesagens das colunas de solo em balança digital e as leituras diárias da constante dielétrica com o equipamento TDR 100. Constatou-se que o modelo polinomial cúbico ajustou-se adequadamente para a estimativa da umidade do solo em função da constante dielétrica para os cinco tipos de solos, destacando um melhor ajuste para os dados do solo de textura média arenosa (franco arenoso), apresentando, baixo valor de RMSE (raiz quadrada da média dos quadrados dos erros), o que sinaliza para o bom desempenho do modelo e apresentou também valor baixo de MEA (média erros absolutos) resultando no melhor ajuste dos dados pela equação e uma melhor eficiência do modelo.
\end{abstract}

Palavras-chave: Constante dielétrica aparente, matriz do solo, porosidade.

\footnotetext{
${ }^{1}$ Eng. Agrônomo, Mestrando em Recursos Hídricos na Universidade Federal de Sergipe, Aracaju, Sergipe, Brasil, e-mail: lucasbaptistaufrb@gmail.com

${ }^{2}$ Eng. Agrícola, Pesquisador da Empresa Brasileira de Pesquisa Agropecuária, Cruz das Almas, Bahia, Brasil, email: eugenio.coelho@embrapa.br

${ }^{3}$ Eng. Agrônomo, Professor Associado IV do Centro de Ciências Agrárias Ambientais e Biológicas da Universidade Federal do Recôncavo da Bahia, Cruz das Almas, Bahia, Brasil, e-mail: fadriano64@gmail.com

${ }^{4}$ Tecnólogo em Irrigação e Drenagem, Doutorando em Engenharia Agrícola na Universidade Federal do Recôncavo da Bahia, Cruz das Almas, Bahia, Brasil, e-mail: mairtong@hotmail.com

${ }^{5}$ Eng. Agrônomo, Professor adjunto do curso de Engenharia Agrícola na Universidade Federal de Sergipe, email: rrgomesfilho@hotmail.com

${ }^{6}$ Eng. Ambiental, Mestre em Recursos Hídricos pela Universidade Federal de Sergipe, Aracaju, Sergipe, Brasil, e-mail: amanda_engambiental@hotmail.com
} 


\title{
CALIBRATION OF ARTISANAL PROBE FOR USE WITH TDR FOR EVALUATION OF SOIL MOISTURES
}

\begin{abstract}
The Time Domain Reflectometry (TDR) has been a very important tool for the quantification of the water content in the soil. However, to use the calibration equipment is necessary to obtain accurate values of volumetric moisture, having in view that there is a difference between the types of materials of the matrix of the soil and porosity. In view of the exposed, this work had as objective to develop and calibrate probes of TDR equipment usage in different soil types. The calibration was performed in laboratory conditions, using soil samples wrapped in PVC columns with volume of $0.0078 \mathrm{~m} 3$. The probes handcrafted with three rods spaced $(0.022 \mathrm{~m})$ and $0.15 \mathrm{~m}$ in length, were installed vertically in the soil columns, with a probe by column. There were the weighings of the soil columns in digital scale and daily readings of the dielectric constant with the TDR equipment 100. It was found that the cubic polynomial model was adjusted properly for the estimation of soil humidity in function of the dielectric constant for the five types of soils, highlighting a best fit for the data of the soil of sandy medium texture (Franco sandy soil), presenting a low value of RMSE (root-mean-square of the errors), which attests to the good performance of the model and also presents low value of MEA (mean absolute errors) resulting in a better data adjustment by the equation and a better efficiency of the model.
\end{abstract}

Keywords: The apparent dielectric constant, matrix of soil, porosity.

\section{INTRODUÇÃO}

O conhecimento do teor de água do solo é imprescindível em estudos que envolva as propriedades do sistema solo-água-planta (COELHO et al., 2006), em processos físicos, químicos e biológicos que ocorrem no solo (MANIERI et al., 2007). A determinação da umidade em solos destinados à produção agrícola é de vital importância para o manejo adequado da água e, consequentemente, da energia para a pressurização dos sistemas de irrigação; por sua vez, o manejo eficiente desses sistemas favorece o desenvolvimento adequado das plantas e contribui para o aumento da produção e preservação do meio ambiente (PEREIRA et al., 2006).

As técnicas de medida da umidade do solo são normalmente classificadas como diretas ou indiretas. Os métodos indiretos determinam alguma propriedade física ou química relacionada à umidade do solo (MANIERI et al., 2007). Dentre estes métodos destaca-se a técnica da Reflectometria no Domínio do Tempo (TDR), é uma das mais exatas. O uso da TDR tem-se difundido na área de pesquisas principalmente por ser um método não-destrutivo, por sua exatidão e pela possibilidade de automação da coleta dos dados (COELHO et al., 2003; COELHO et al., 2005; COELHO et al., 2006).

Segundo Coelho et al. (2001) e Coelho et al. (2005), diversos tipos de TDR têm sido disponibilizados no mercado, porém uma preocupação constante na utilização do equipamento é com as sondas fornecidas pelo fabricante do dispositivo, pois os preços dessas guias de onda, associados aos custos de importação, têm sido limitantes para o uso desses equipamentos.

Visando reduzir os custos na aquisição das sondas utilizadas nos equipamentos TDR, pesquisas no Brasil têm sido conduzidas com o objetivo de confeccionar as sondas artesanalmente (COELHO et al., 2001; ANDRADE et al., 2003; COELHO et al., 2003; COELHO et al., 2005; SOUZA et al., 2006; MILANI et al., 2008; ELAIUY et al., 2009; SANTOS et al., 2010; SONCELA et al., 2011; SANT'ANA et al., 2012; SONCELA et al., 2013).

O princípio de funcionamento da técnica TDR baseia-se no tempo de percurso de um 
pulso eletromagnético entre as hastes de uma sonda inserida no solo, o qual é dependente da constante dielétrica média do meio (média ponderada das constantes dielétricas das frações das fases sólida, líquida e gasosa) (SANTOS et al., 2012). A partir dos valores da constante dielétrica do solo, correlaciona-se aos dados de conteúdo de água por meio de curvas de calibração obtidas a partir dos dados de campo ou laboratório (ALMEIDA et al., 2012).

Tal equipamento necessita de calibração para que se obtenha o valor correto da umidade volumétrica, devendo-se respeitar as peculiaridades, como, por exemplo, os altos teores de ferro e argila e a expansibilidade do solo (VILLWOCK et al., 2004).

No intuito de resolver o problema de calibração do equipamento TDR, equações como a de Topp et al. (1980) foram criadas e utilizadas para se estimar o conteúdo de água no solo. Essa equação apresenta uma relação entre umidade e constante dielétrica aparente do solo demasiadamente generalista, que poderia ser utilizada para uma ampla diversidade de solos, desconsiderando-se praticamente toda a influência pertinente aos demais componentes do solo que concorrem para a constante dielétrica aparente, tais como sais, textura e densidade (SILVA, 2009).

Porém, o modelo proposto de Topp et al. (1980) não possui aplicabilidade universal, uma vez que alguns atributos do solo podem conferir erros às medições (SILVA, 1998; CICHOTA \& LIER, 2002; TOMMASELLI, 1997, entre outros). Por exemplo, em solos tropicais, nos quais o elevado teor de óxido de ferro frequentemente constitui-se como fontes significativas de erros (TOMMASELLI, 1997). Coelho et al. (2006) ressaltam que os modelos de calibração variam conforme o tipo de solo e que um modelo polinomial de terceiro grau pode ser calibrado com exatidão para cada situação de solo.

Assim, surge a necessidade de ajuste de equações para cada tipo de solo (VILLWOCK et al., 2004; LACERDA et al., 2005; COELHO et al., 2006; PEREIRA et al., 2006; MEDEIROS et al., 2007; MILANI et al., 2008; BIZARI et al., 2011; SANTOS et al., 2012; SOUZA et al., 2013; CARVALHO et al., 2015).

O presente trabalho objetivou calibrar e avaliar as sondas artesanais de uso em equipamentos TDR, para estimativa do conteúdo de água em cinco tipos de diferentes solos, em condições de laboratório.

\section{MATERIAL E MÉTODOS}

O presente trabalho foi conduzido no Laboratório de Irrigação e Fertirrigação da Embrapa Mandioca e Fruticultura Tropical, em Cruz das Almas (12 48' S; 39 06’ W e 225 m). Foram coletadas amostras de cincos solos de texturas diferentes, classificados de acordo as premissas da Embrapa (2006) e cujas características físicas (Tabela 1) foram determinadas conforme metodologia recomendada pela Embrapa (1997).

Tabela 1. Características físicas dos solos utilizados no trabalho.

\begin{tabular}{lccc}
\hline \multirow{2}{*}{ Classe textural } & \multicolumn{3}{c}{ Composição granulométrica $\left(\mathrm{g} / \mathrm{kg}^{-1}\right)$} \\
\cline { 2 - 4 } & Areia & Silte & Argila \\
\hline Areia franca & 858 & 72 & 70 \\
Areia & 885 & 95 & 20 \\
Argila & 196 & 392 & 412 \\
Franco arenoso & 528 & 371 & 101 \\
Franco argilo arenoso & 510 & 238 & 252 \\
\hline
\end{tabular}

As amostras dos cinco tipos de solos deformadas foram destorroadas e secas ao ar sendo, em seguida, acondicionadas em tubos de PVC, previamente pesados, de diâmetro de 0,075 $\mathrm{m}$ e altura de $0,20 \mathrm{~m}$. Usou-se uma tela fina previamente pesada, que foi colada à parede da parte inferior do segmento de tubo para evitar perda de solo. O solo dentro dos segmentos de tubo foi saturado durante $48 \mathrm{~h}$. 
Foram construídas guias de onda de TDR artesanalmente, com três hastes de aço inoxidável. As sondas (guias de onda) foram construídas para atender ao equipamento de TDR 100, da Campbell Scientific, e serem acopladas diretamente ao equipamento. As sondas se compunham de três hastes de 0,15 m, espaçadas 0, 022 m (SONCELA et al., 2013), à semelhança das construídas para atender aos equipamentos Tektroniks ou Trase System (COELHO et al., 2001; SOUZA et al., 2001). Na construção de uma sonda de três hastes para uso com reflectômetros, requer materiais como: catalisador de resina, solda elétrica, ácido fosfórico 1\%, e alicate de clipagem (COELHO et al., 2005; SANT'ANA et al., 2012).

As guias de onda foram pesadas e inseridas no solo úmido durante a saturação. Após a instalação das antenas, os recipientes foram mantidos com a superfície do solo exposta, para permitir perda de água apenas por evaporação. As colunas de solo com as guias de ondas de TDR foram sucessivamente pesadas a partir da saturação e ficaram expostas no laboratório até ser atingido o limite inferior de umidade disponível. As leituras de umidade foram feitas com diariamente com a TDR, seguidas de pesagens das colunas. Quando a umidade do solo das colunas atingiu valores próximos do limite inferior da disponibilidade de água, as colunas foram levadas à estufa a $105^{\circ} \mathrm{C}$ até atingirem massa constante. Durante cada pesagem, foi feita a leitura no equipamento de TDR, obtendo-se o tempo de propagação do pulso eletromagnético e, consequentemente, a constante dielétrica aparente do solo, da qual indiretamente se estimou a umidade volumétrica $\left(\theta_{\mathrm{i}}\right)$ do solo (Equação 1).

$$
\theta_{\mathrm{i}}=\frac{\mathrm{PU}-\mathrm{PS}}{\mathrm{V}}
$$

em que: PU é o peso do solo úmido atual $+\mathrm{PE}$, em g; PS é o peso do solo seco em estufa $+\mathrm{PE}$, em g; PE é o peso da coluna de PVC + peso da guia de onda + duas telas de náilon, em g; V é o volume do solo, em $\mathrm{cm}^{3}$.

O conteúdo de água no solo $\left(\theta_{\mathrm{v}}\right)$ foi estimado por meio da equação de Ledieu et al. (1986) (Equação 2) segundo Santana et al.
(2006) e, posteriormente, corrigido de acordo o modelo polinomial cúbico (Equação 3), por apresentar bom desempenho em trabalhos anteriores (COELHO et al., 2006; MILANI et al., 2008; ELAIUY et al., 2009; SANT'ANA et al., 2012; SANTOS et al., 2012).

$$
\begin{aligned}
& \theta_{v}=0,1138 \sqrt{K a}-0,1758 \\
& \theta_{v}=a+b K a+c K a+d K a
\end{aligned}
$$

em que: $\theta_{\mathrm{v}}$ - Conteúdo de água no solo, $\mathrm{cm}^{3} \mathrm{~cm}^{-}$ ${ }^{3}$; $\mathrm{Ka} \mathrm{-} \mathrm{constante} \mathrm{dielétrica} \mathrm{do} \mathrm{solo,}$ adimensional.

Os valores de umidade volumétrica estimada pelo modelo de calibração das sondas de uso em TDR foram comparados por meio de regressão linear simples, do tipo $\mathrm{y}=\mathrm{bx}$, em que o coeficiente angular próximo de 1,0, com coeficiente de determinação $\left(\mathrm{R}^{2}\right)$ elevado indicando maior precisão do modelo ajustado. Os indicadores estatísticos da calibração foram: RMSE (raiz quadrada da média dos quadrados dos erros) (Equação 3), MEA (média dos erros) (Equação 4) e a eficiência do modelo de calibração (Equação 5).

$$
\begin{aligned}
& \text { RMSE }=\sqrt{\frac{1}{n} \sum_{i=1}^{n}\left(O_{i}-E_{i}\right)^{2}} \\
& M E A=\frac{1}{n} \sum_{i=1}^{n}\left(O_{i}-E_{i}\right)^{2} \\
& \operatorname{Ef}=\frac{\left[\sum_{i=1}^{n}\left(O_{i}-O\right)-\sum_{i=1}^{n}\left(O_{i}-E_{i}\right)^{2}\right]}{\sum_{i=1}^{n}\left(O_{i}-O\right)^{2}}
\end{aligned}
$$

em que: $\mathrm{O}_{\mathrm{i}}$ é a umidade volumétrica observada, $\mathrm{cm}^{3} \mathrm{~cm}^{-3}$; O é a média da umidade volumétrica observada, $\mathrm{cm}^{3} \mathrm{~cm}^{-3} ; \mathrm{E}_{\mathrm{i}}$ é a umidade volumétrica estimada, $\mathrm{cm}^{3} \mathrm{~cm}^{-3}$; $\mathrm{n}$ é o número de dados observados.

\section{RESULTADOS E DISCUSSÃO}


Na Figura 2 é apresentado o ajuste do modelo polinomial cúbico para determinação da umidade volumétrica em função da constante dielétrica relativa aparente para os cinco tipos de solos.

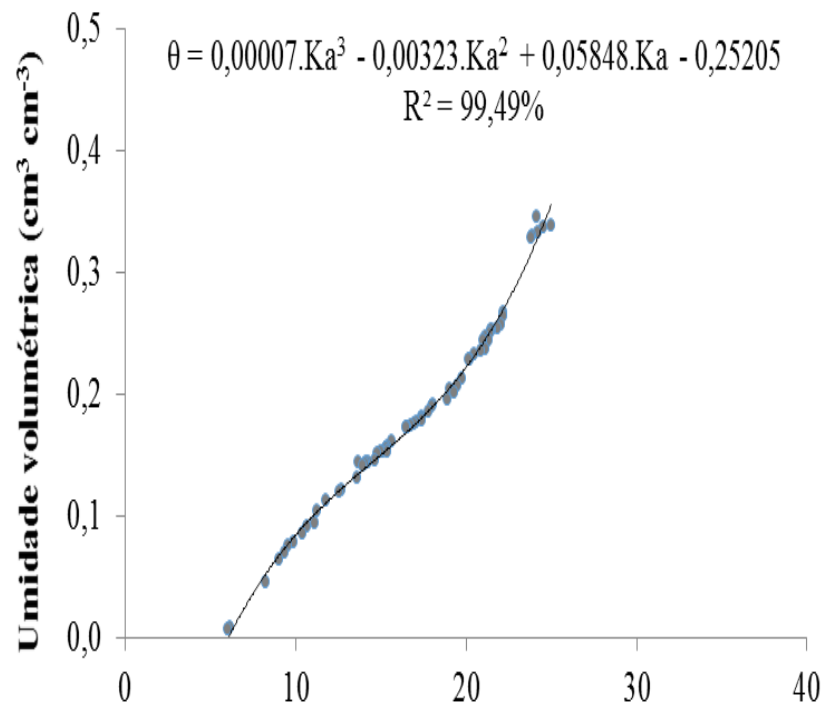

(A) Constante dielétrica relativa aparente $(\mathrm{Ka})$

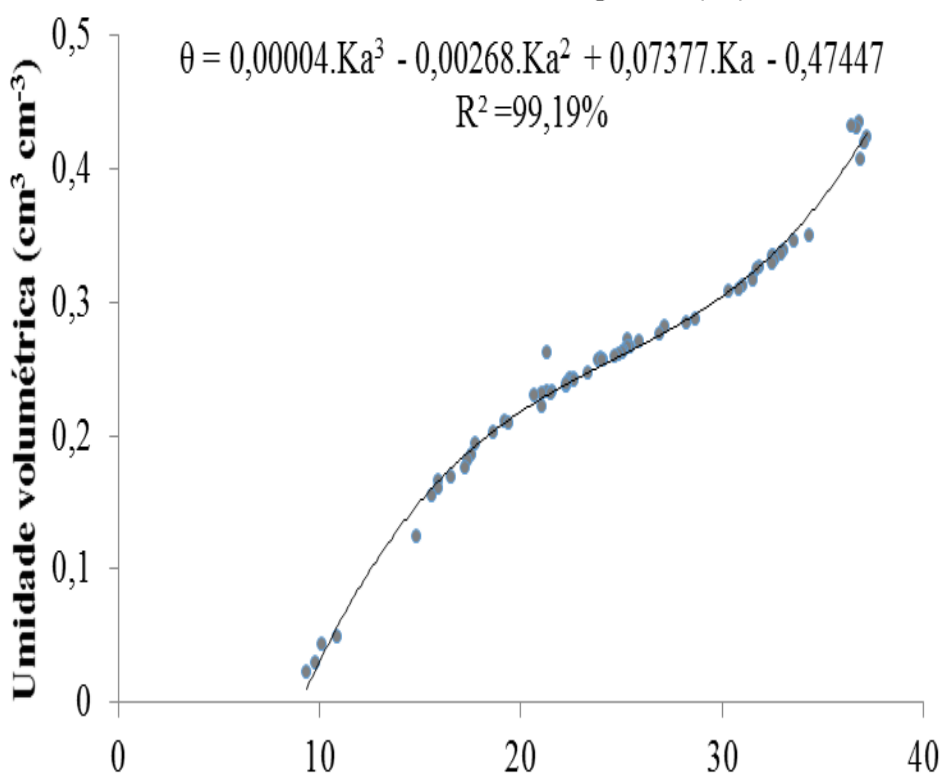

(C)

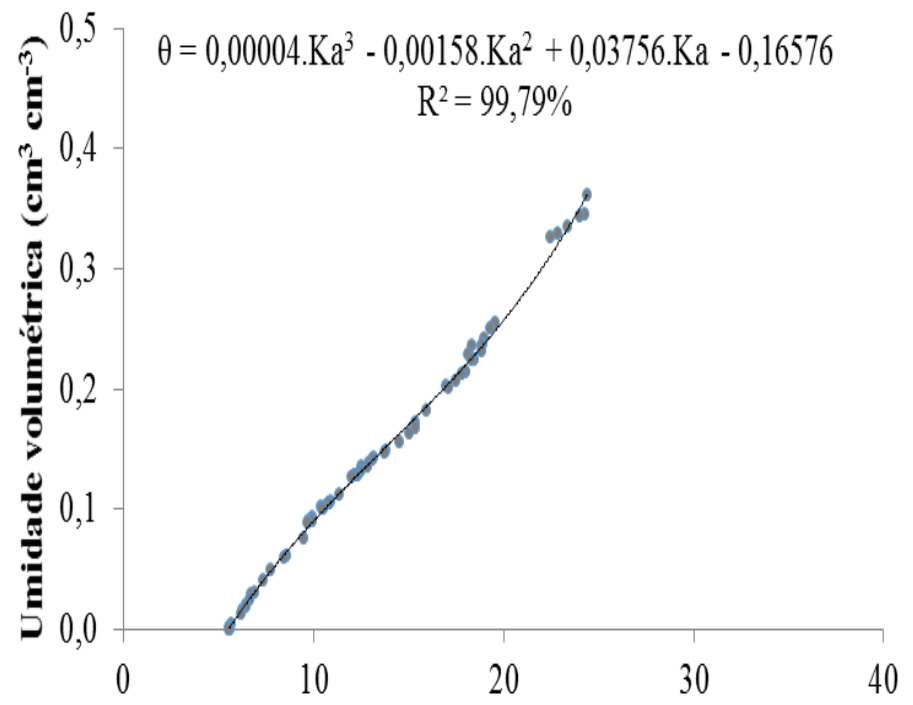

(B)

Constante dielétrica relativa aparente $(\mathrm{Ka})$

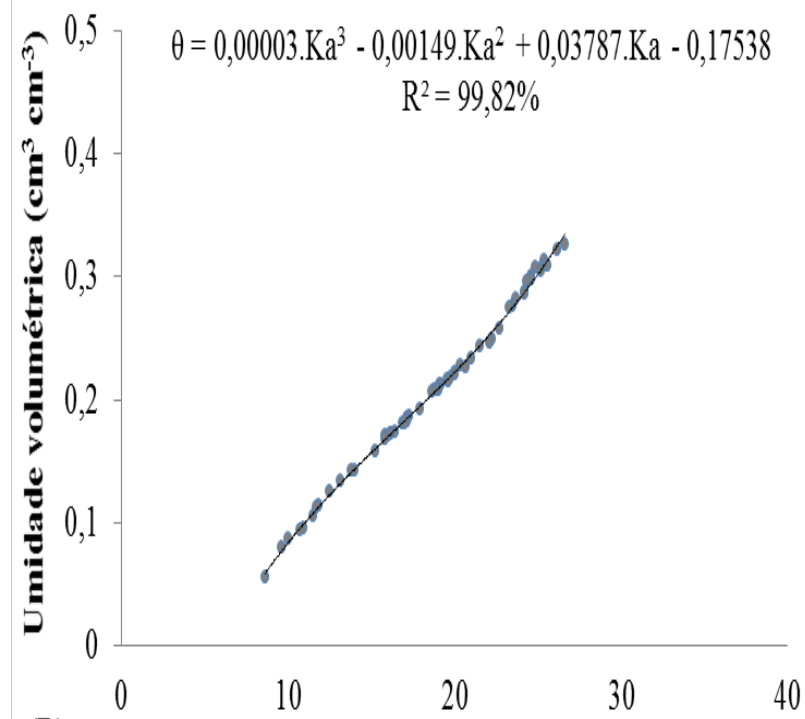

(D) Constante dielétrica relativa aparente (Ka) 


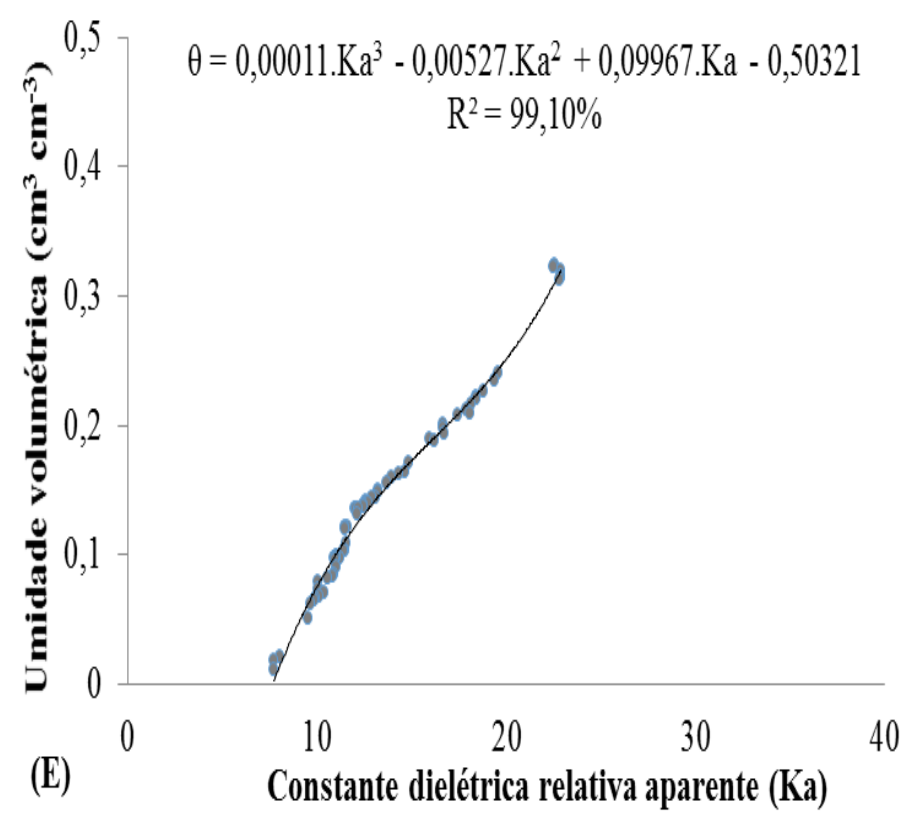

Figura 2. Curvas de calibração para diferentes tipos de solos, areia franca (A), areia (B), argila (C), franco arenoso (D) e franco argilo arenoso (E).

Todas as curvas de calibração apresentaram coeficiente de determinação próximo da unidade $(1,0)$. Com base em trabalhos anteriores, tem-se justificado o uso do modelo cúbico para diferentes tipos de solos, como por exemplo, o trabalho de Tommaselli (1997), em que o modelo polinomial cúbico foi superior a outros na estimativa do conteúdo de água no solo em função da constante dielétrica para solos com textura argilosa. De acordo com os resultados reportados por Pereira et al. (2006), o modelo cúbico apresentou-se como o melhor ajuste para o solo de textura mais grossa. Para Coelho et al. (2006), os modelos de calibração variam conforme o tipo de solo e que um modelo polinomial de terceiro grau pode ser calibrado com exatidão para cada situação de solo. Justificando isso, Santos et al. (2010) relatou ser evidente que, além da textura, os componentes químicos de cada solo podem ocasionar determinado comportamento da equação de calibração.
Os resultados dos indicadores estatísticos da calibração (Tabela 2) demonstram que, em geral, a calibração para todos os tipos apresentaram um baixo valor de RMSE, o que sinaliza para o bom desempenho do modelo testado. Segundo Gumiere et al. (2006), o RMSE mede basicamente o deslocamento do erro em relação ao erro médio, o valor ideal é zero, entretanto como todos os modelos trabalham com aproximações é muito difícil encontrar o valor zero para o RMSE. Assim, em geral, quanto menor o valor do RMSE melhor o desempenho do modelo de calibração utilizado. Consequentemente os valores da MEA (média absoluta dos erros) são baixos, resultando em melhor ajuste dos dados pela equação. Quanto à eficiência do modelo, os solos de textura média a arenosa apresentaram valores próximos da unidade $(1,0)$, o que sinaliza para o bom desempenho do modelo cúbico. Já o solo argiloso apresentou uma menor eficiência no presente modelo.

Tabela 2. Avaliação das equações de calibração obtidas para os diferentes tipos de solo, com base na raiz quadrada da média dos quadrados dos erros (RMSE), média absoluta dos erros (MAE) e na eficiência do modelo (EF). 


\begin{tabular}{lccc}
\hline Areia franca & 0,032753712 & $1,07 \mathrm{E}-03$ & 0,8380 \\
Areia & 0,012722473 & 0,0001619 & 0,9812 \\
Argila & 0,549968609 & 0,008292 & 0,7754 \\
Franco arenoso & 0,005161538 & $2,66 \mathrm{E}-05$ & 0,9944 \\
Franco argilo arenoso & 0,008465841 & $7,17 \mathrm{E}-05$ & 0,9877 \\
\hline
\end{tabular}

Os resultados encontrados demonstraram existir viabilidade no uso das sondas de TDR propostas como alternativa às comerciais na estimativa da umidade do solo. Isto concorda com os relatos de Souza et al. (2006), pois segundo aqueles autores, as sondas desenvolvidas artesanalmente possuem características físicas adequadas, viabilizando a utilização da técnica nas mais diversas condições, principalmente em situações que necessitam de quantidade elevada de sondas de TDR.

Em concordância com os indicadores de eficiência dos modelos, os solos de textura arenosa apresentaram melhor calibração da sonda de uso em TDR, uma vez que os coeficientes angulares foram mais próximos da unidade

(Figura

3).

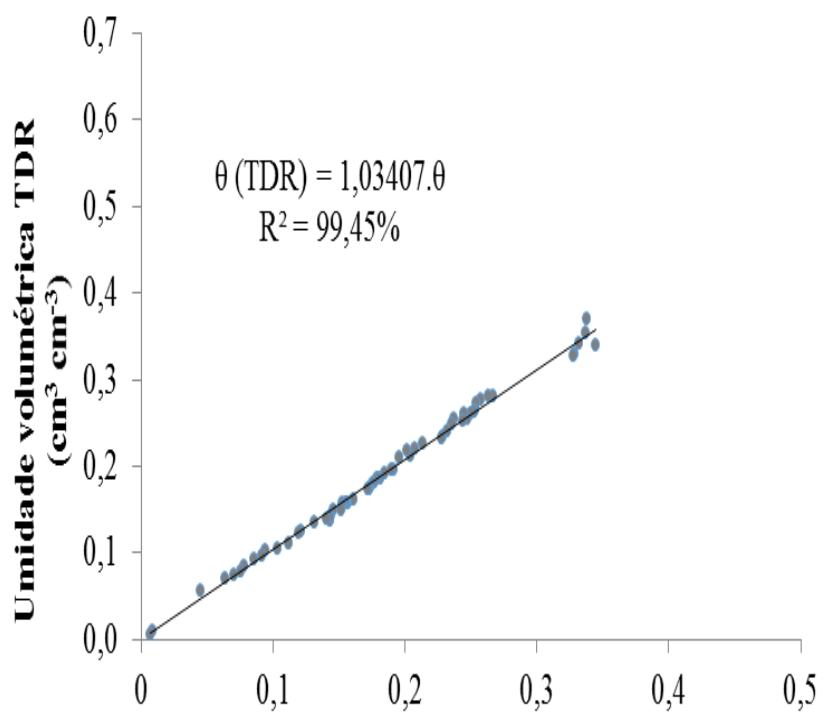

(A)

Umidade volumétrica $\left(\mathrm{cm}^{3} \mathrm{~cm}^{-3}\right)$

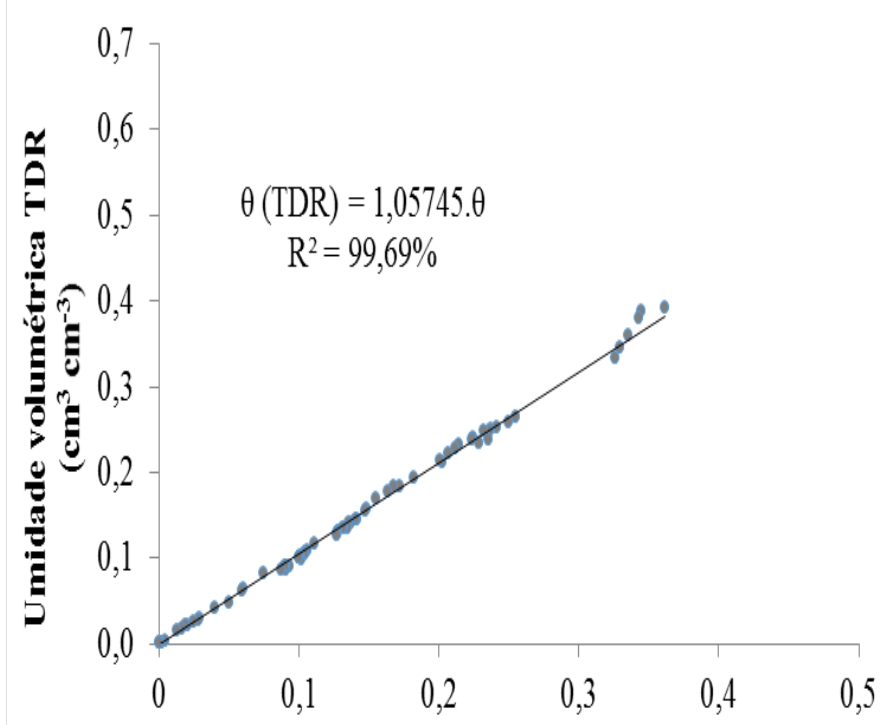

(B)

Umidade volumétrica $\left(\mathrm{cm}^{3} \mathrm{~cm}^{-3}\right)$ 

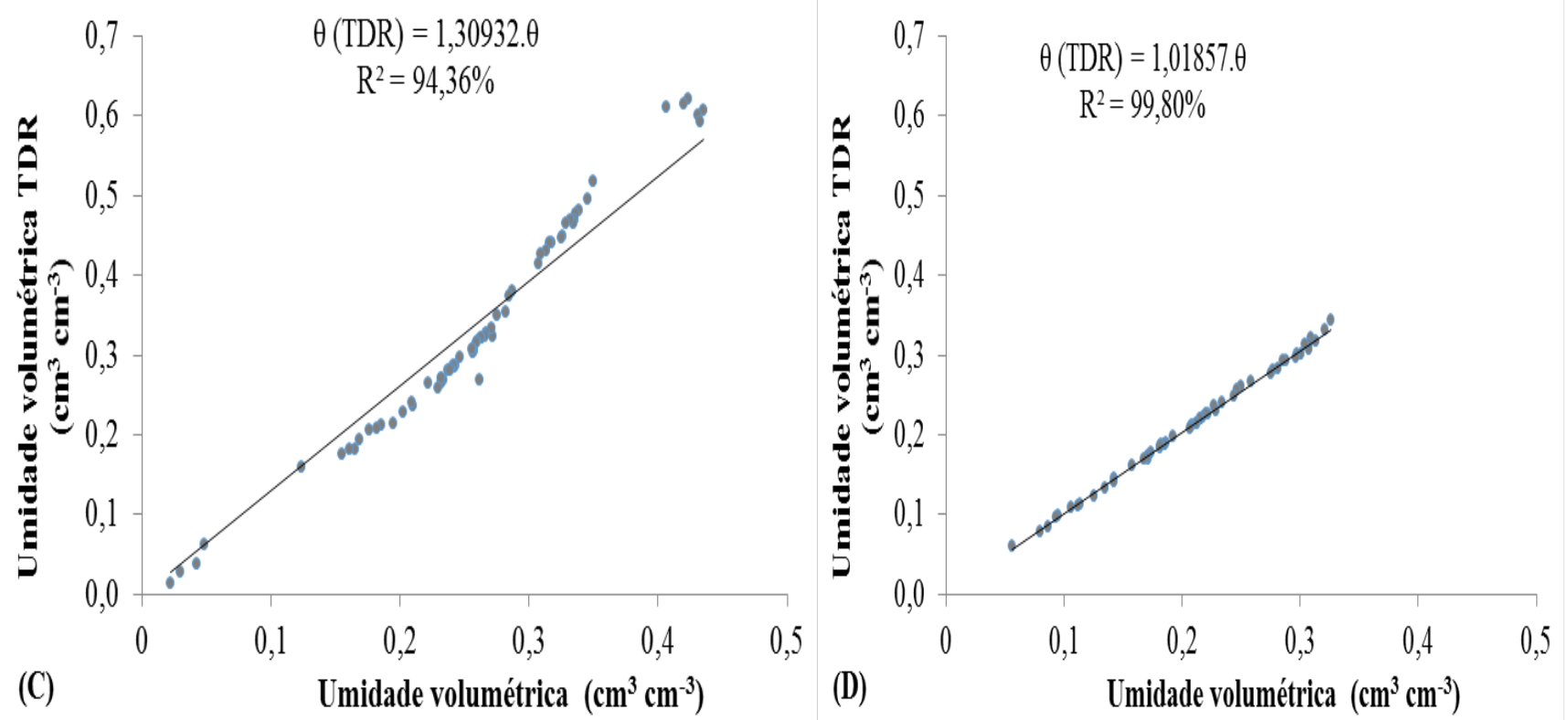

(D)

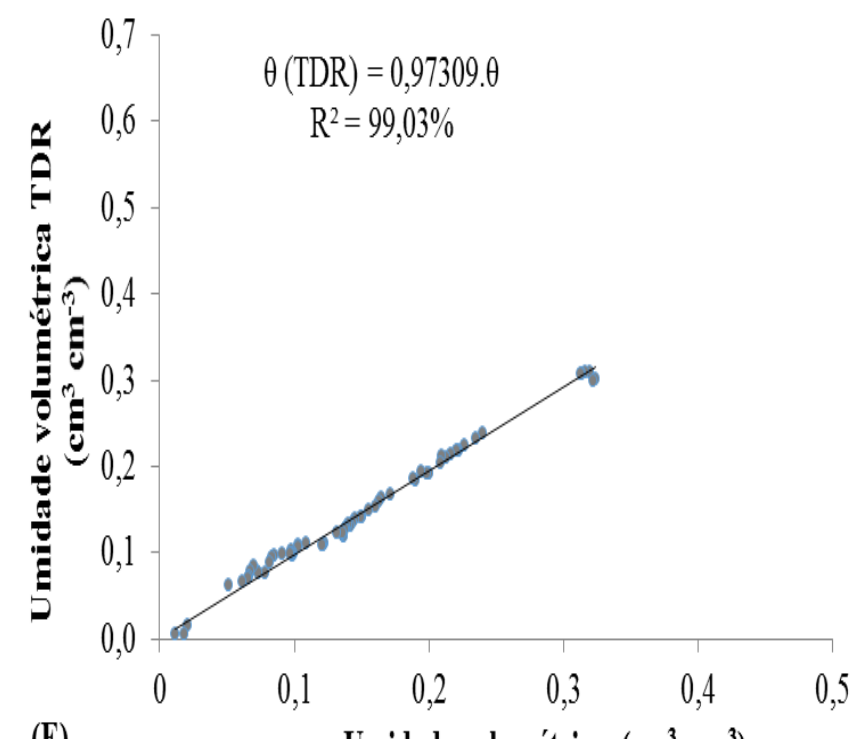

(E)

Umidade volumétrica $\left(\mathrm{cm}^{3} \mathrm{~cm}^{-3}\right)$

Figura 3. Intercepto em zero dos valores estimados e medidos de umidade volumétrica para diferentes tipos de solos, areia franca (A), areia (B), argila (C), franco arenoso (D) e franco argilo arenoso (E).

Sendo o solo franco arenoso que melhor se adequo ao modelo, subestimando a umidade simulada em relação à umidade medida em $1,8 \%$. Sendo esse resultado contrário ao encontrado por Trintinalha et al. (2004), pois em um solo de textura muito argilosa obtiveram o melhor ajuste uma equação descrita por uma função linear. Segundo Fagundes et al. (2011), o conhecimento dessas equações é essencial para a implementação de sistemas de irrigação que utilizam-se de programas para leitura e armazenamento de dados, que ligados aos sensores farão a leitura dos dados de umidade do solo, minimizando os possíveis erros.

\section{CONCLUSÕES}

As equações de calibração obtidas explicaram satisfatoriamente a variação da umidade volumétrica para os cinco tipos de solo.

As sondas de uso em equipamento TDR feitas com material nacional de baixo custo são apresentadas como uma alternativa viável para a obtenção de conteúdo de água no solo.

\section{REFERÊNCIAS BIBLIOGRÁFICAS}

ALMEIDA, C. A. B.; ANTONINO, A. C. D.; PIMENTEL, R. M. M.; LIRA, C. A. B. O.; 
LIMA, J. R. S. Influência da densidade na estimativa da umidade volumétrica em um Latossolo Vermelho-Amarelo. Revista Brasileira de Geografia Física, v.5, n.5, p.1056-1068, 2012.

ANDRADE, C. L. T.; COSTA, E. L.; ALBUQUERQUE, E. P. Desenvolvimento e calibração de guias de onda para TDR. Revista Brasileira de Engenharia Agrícola e Ambiental, v.7, n.1, p.173-176, 2003.

BIZARI, D. R.; MATSURA, E. E.; SOUZA, C. F.; ROQUE, M. W. Haste portátil para utilização de sondas de TDR em ensaios de campo. Irriga, v.16, n.1, p.31-41, 2011.

CARVALHO, T. R. A.; MASSARANDUBA, W. M.; FONTENELE, S. B.; LANDIM, R. B. T. V.; MENDONÇA, L. A. R. Ajuste de modelo para determinar a umidade em Neossolo Flúvico com uso de uma sonda Time Domain Reflectometry (TDR). Revista Agrarian, v.8, n.27, p.57-63, 2015.

COELHO, E. F.; ANDRADE, C. L. T.; OR, D.; LOPES, L. C.; SOUZA, C. F. Desempenho de diferentes guias de ondas para uso com o analisador de umidade TRASE. Revista Brasileira de Engenharia Agrícola e Ambiental, v.5, n.1, p.81-87, 2001.

COELHO, E. F.; CALDAS, R. C.; SANTOS, D. B.; LEDO, C. A. S. Número e espaçamento entre hastes de guia de onda para medida da umidade do solo com TDR. Revista Brasileira de Engenharia Agrícola e Ambiental, v.7, n.2, p.215-220, 2003.

COELHO, E. F.; VELLAME, L. M.; COELHO FILHO, M. A. Sonda de TDR para estimativa da umidade e condutividade elétrica do solo, com uso de multiplexadores. Revista Brasileira de Engenharia Agrícola e Ambiental, v.9, n.4, p.475-480, 2005.

COELHO, E. F.; VELLAME, L. M.; COELHO FILHO, M. A.; LEDO, C. A. S. Desempenho de modelos de calibração de guias de onda acopladas a TDR e a multiplexadores em três tipos de solos. Revista Brasileira de Ciência do Solo, v.30, n.1, p.23-30, 2006.

CICHOTA, R.; LIER, Q. J. V. Calibração no campo de um equipamento de TDR segmentado. In: REUNIÃO BRASILEIRA DE MANEJO E CONSERVAÇÃO DO SOLO E DA ÁGUA, 14., 2002, Cuiabá. Anais... Cuiabá: SBCS/UFMT. 1CDROM.

ELAIUY, M. L. C.; SATO, L. M.; VARALHO, A. C. T.; SOUZA, C. F. Desenvolvimento e avaliação de sonda TDR para o manejo racional da água em substratos utilizados na produção de mudas florestais. Revista Ambiente \& Água, v.4, n.1, p.117-131, 2009.

EMBRAPA. Centro Nacional de Pesquisa de Solos. Manual de métodos de análise de solo. 2.ed. Rio de Janeiro, 1997. 212p.

EMBRAPA - Empresa Brasileira de Pesquisa Agropecuária. Centro Nacional de Pesquisa de Solos. Sistema brasileiro de classificação de solos. Rio de Janeiro, 2006.

FAGUNDES, M. C.; MORAES, M. O.; SCHAFFER, D.; QUEIROZ, T. M. Calibração de sensores para determinação do teor de água do solo. Revista de Ciências Agroambientais, v.9, n.1, p.53-58, 2011.

GUMIERE, S. J.; MORAS, J. M.; VICTÓRIA, R. L. Calibração e validação de um modelo solovegetação-atmosfera na Floresta Nacional de Tapajós, Pará. Revista Brasileira de Recursos Hídricos, v.11, n.4, p. 25-35, 2006.

LACERDA, R. D.; GUERRA, H. O. C.; BARROS JUNIOR, G.; CAVALCANTI, M. L. F. Avaliação de um TDR para determinação do conteúdo de água do solo. Revista de Biologia e Ciências da Terra, v.5, n.1, p.1-12, 2005.

LEDIEU, J.; DE RIDDER, P.; DE CLERCK, P.; DAUTREBANDE, S. A method measuring soil water moisture by time-domain reflectometry. Journal of Hydrology, v.88, n.1, p.319-28, 1986. 
MANIERI, J. M.; VAZ, C. M. P. V.; MARIA, I. C. M. Sonda espiral de TDR para a medida da umidade no perfil do solo. Revista Brasileira de Ciência do Solo, v.31, n.2, p.191-198, 2007.

MEDEIROS, J. D. F.; CASTRO, N.; GOLDENFUM, J. A.; CLARKE, R. T. Calibração de sondas TDR em um Latossolo. Revista Brasileira de Recursos Hídricos, v.12, n.2, p.19-25, 2007.

MILANI, D. I. C.; TAVARES, M. H. F. CLEMENTINA, S. Calibração de sondas TDR em condições de laboratório. Revista Ambiente \& Água, v.3, n.1, p.68-75, 2008.

PEREIRA, S.; OLIVEIRA FILHO, D.; MANTOVANI, E. C.; RAMOS, M. M.; MARTINS, J. H. Reflectometria no domínio do tempo na determinação do conteúdo de água no solo. Revista Brasileira de Engenharia Agrícola e Ambiental, v.10, n.2, p.306-314, 2006.

SANTANA, G. S.; COELHO, E. F.; SILVA, T. S. M.; RAMOS, M. M. Estimativa da condutividade elétrica da solução do solo a partir do teor de água e da condutividade elétrica aparente do solo. Engenharia Agrícola, v.26, n.3, p.672-685, 2006.

SANT'ANA, J. A. V.; COELHO, E. F.; SILVA JUNIOR, J. J.; SANTANA JUNIOR, E. B.; VEINROMBER JUNIOR, L. A. A. Desempenho de sondas de TDR manufaturadas de diferentes comprimentos de hastes. Revista Brasileira de Ciência do Solo, v.36, n.4, p. 1123-1130, 2012.

SANTOS, D. B.; CECÍLIO, R. A.; COELHO, E. F.; BATISTA, R. O.; SILVA, A. J. P. Calibração de TDR: desempenho de alguns métodos e equações de calibração. Revista Agrarian, v.5, n.16, p.131-139, 2012.

SANTOS, M. R.; ZONTA, J. H.; MARTINEZ, M. A. Influência do tipo de amostragem na constante dielétrica do solo e na calibração de sondas de TDR. Revista Brasileira de Ciência do Solo, v.34, n.2, p.299-308, 2010.
SILVA, E. L. Determinação automática do teor de água em Latossolo roxo distrófico com uso de reflectometria de onda. In: XXVII CONGRESSO BRASILEIRO DE ENGENHARIA AGRÍCOLA, 27., Poços de Caldas. Anais... Poços de Caldas: UFLA/SBEA, 1998. p. 154-156.

SILVA, G. F.; MOLIN, J. P.; DIAS, C. T. S. Equação de calibração de um TDR comercial para determinação do teor de água em solos de Piracicaba-SP. 2009. 10p. Monografia (Graduação em Engenharia Agronômica) Escola Superior de Agricultura "Luiz de Queiroz", Universidade de São Paulo, Piracicaba-SP.

SONCELA, R.; SAMPAIO, S. C.; VILAS BOAS, M. A.; TAVARES, M. H. F.; SOUZA, C. F.; SONCELA, A. S. Electrical conductivity of soil irrigated with swine wastewater estimated by time-domain reflectometry. Revista Brasileira de Ciência do Solo, v.35, n.4, p.1293-1300, 2011.

SONCELA, R.; SAMPAIO, S. C.; VILAS BOAS, M. A.; TAVARES, M. H. F.; SMANHOTTO, A. Construction and calibration of TDR probes for volumetric water content estimation in a distroferric red latosol. Engenharia Agrícola, v.34, n.5, p.919-928, 2013.

SOUZA, C. F.; MATSURA, E. E.; TESTEZLAF, R. Experiência de laboratório de hidráulica, irrigação e drenagem da Faculdade de Engenharia Agrícola/UNICAMP no uso da técnica da TDR. In: MATSURA, E. E.; JAVAREZ JUNIOR, A.; GOMES, E. P.; SOUZA, C. F. (Eds.). Aplicações técnicas de TDR na agricultura. Campinas: FEAGRI/UNICAMPI, 2001. p.147-176.

SOUZA, C. F.; MATSURA, E. E.; FOLEGATTI, M. V.; COELHO, E. F.; OR, D. Sondas de TDR para a estimativa da umidade e da condutividade elétrica do solo. Irriga, v.11, n.1, p.12-25, 2006. 
SOUZA, C. F.; PIRES, R. C. M.; MIRANDA, D. B.; VARALLO, A. C. T. Calibração de sondas FDR e TDR para a estimativa da umidade em dois tipos de solo. Irriga, v.18, n.4, p.597606, 2013.

TOMMASELLI, J. T. G. Influência de algumas características do solo sobre a calibração de um equipamento de TDR (Time-Domain Reflectometry). 1997. 109p. Tese (Doutorado Energia Nuclear na Agricultura) - Escola Superior de Agricultura "Luiz de Queiroz", Universidade de São Paulo, Piracicaba.

TOPP, G. C.; DAVIS, J. L.; ANNAN, A. P. Electromagnetic determination of soil water content: measurements in coaxial transmission lines. Water Resources Research, v.16, p.574582, 1980.

TRINTINALHA, M. A.; GONÇALVES, A. C. A.; TORMENA, C. A.; COSTA, A. C. S.; FOLEGATTI, M. V.; FREITAS, P. S. L.; REZENDE, R. Comparação dos sistemas TDR e ECHO para medida de umidade, em um solo argiloso e em areia. Acta Scientiarum: Agronomy, v.26, n.3, p.353-360, 2004.

VILLWOCK, R.; TAVARES, M. H. F.; VILAS BOAS, M. A. Calibração de um equipamento TDR em condições de campo. Irriga, v.9, n.1, p.82-88, 2004. 